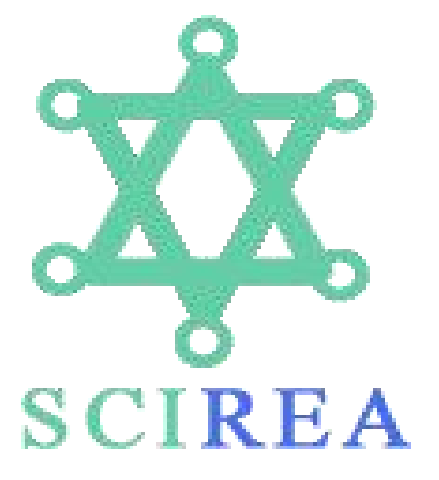

SCIREA Journal of Physics

ISSN: 2706-8862

http://www.scirea.org/journal/Physics

February 12, 2022

Volume 7, Issue 1, February 2022

https://doi.org/10.54647/physics 14403

\title{
The Photon according to Einstein
}

\author{
Richard Oldani \\ Retired \\ Email: oldani@juno.com
}

\begin{abstract}
The primary content of this paper consists of direct quotes from Einstein, arguments about the nature of the photon from over 100 years ago that have been quietly ignored because they are not in conformance with interpretations of quantum mechanics according to the standard model. Although faulted for his disagreements with quantum theory, we show here that once again his intuitive reasoning, as derived from the fundamentals, is perfect. After carefully analyzing the foundations of quantum theory and electromagnetic wave theory during the years 1906 - 1909 he hypothesized that the photon' s structure is similar to that of an electron; a discrete entity with particle properties at its core surrounded by continuous fields that superpose linearly. By following his suggestions we are able to describe a field model that satisfies the requirements of the classical, relativistic, and quantum theories.
\end{abstract}

Keywords: photon, radiation, light, wave-particle duality, Maxwell's equations, quantum mechanics 


\section{Introduction}

It was 1909 when in a lecture to the German Physical Society Einstein proclaimed that a theory of radiation was needed in which wave and particle properties are equally prominent [1] "There is an extensive group of facts concerning radiation that shows that light possesses certain fundamental properties that can be understood far more readily from the standpoint of Newton's emission theory of light than from the standpoint of the wave theory. It is therefore my opinion that the next stage in the development of theoretical physics will bring us a theory of light that can be understood as a kind of fusion of the wave and emission theories of light. To give reasons for this opinion and to show that a profound change in our views on the nature and constitution of light is imperative is the purpose of the following remarks."

\section{Theoretical model of the photon [1]}

During the lecture he gave extensive theoretical and experimental evidence from his earlier work in special relativity [2], mass-energy equivalence [3], Planck's radiation law, and the photoelectric effect [4] to demonstrate the need for a relativistic theory, a theory of radiation that includes the photon. Although unable to obtain a photon model that can account for the observed wave and particle properties of light he summarized his studies by suggesting the following possibilities for its structure.

"Still, for the time being the most natural interpretation seems to me to be that the occurrence of electromagnetic fields of light is associated with singular points just like the occurrence of electrostatic fields according to the electron theory. It is not out of the question that in such a theory the entire energy of the electromagnetic field might be viewed as localized in these singularities, exactly like in the old theory of action at a distance. I more or less imagine each such singular point as being surrounded by a field of force which has essentially the character of a plane wave and whose amplitude decreases with the distance from the singular point. If many such singularities are present at separations that are small compared with the dimensions of the field of force of a singular point, then such fields of force will superpose, and their totality will yield an undulatory field of force that may differ only slightly from an undulatory field as by the current electromagnetic theory of light. I am sure it need not be particularly emphasized that no importance should be attached to such a picture as long as it has not led to an exact theory. All I wanted is briefly to indicate with its help that the two structural properties (the undulatory structure and the quantum structure) simultaneously 
displayed by radiation according to the Planck formula should not be considered as mutually incompatible."

\section{Discussion following Einstein's lecture [5]}

Max Planck, acting as moderator, summarized the problems that would have to be overcome for a quantum theory of light, but he opposed a theory of the type Einstein suggested that would include photons, preferring instead the classical theory of Maxwell as an alternative.

Planck: "I think that first of all one should attempt to transfer the whole problem of the quantum theory to the area of interaction between matter and radiation energy; the processes in pure vacuum could then temporarily be explained with the aid of the Maxwell equations."

Stark: "Mr. Planck pointed out that we have no reason at the moment to switch over to the Einstein consequence, to consider the radiation in space, where it occurs detached from matter, as concentrated. I was originally also of the opinion that for the time being we could restrict ourselves to reducing the elementary law to a certain mode of action of the resonators. But I do believe that there exists a phenomenon that leads to the conclusion that electromagnetic radiation detached from matter, in space, must be considered as concentrated. I have in mind the phenomenon that even at great distances, up to $10 \mathrm{~m}$, electromagnetic radiation that has left an X-ray tube for the surrounding space can still achieve concentrated action on a single electron. I believe that this phenomenon does represent a reason for considering the question of whether the energy of electromagnetic radiation should not be considered as concentrated even where it occurs detached from matter."

Planck: "Stark brought up something in favor of the quantum theory, and I wish to bring up something against it; I have in mind the interferences at the enormous phase differences of hundreds of thousands of wavelengths. When a quantum interferes with itself, it would have to have an extension of hundreds of thousands of wavelengths. This is also a certain difficulty."

Stark: "The interference phenomena can easily be pitted against the quantum hypothesis. However, once they are treated with more benevolence toward the quantum hypothesis, one will find an explanation for them, too - this is my hope. As for the experimental aspect, it must be emphasized that the experiments to which Mr. Planck alluded involve very dense radiation, 
so that a very large number of quanta of the same frequency were concentrated in the beam of light; this must be taken into account when discussing those interference phenomena. With radiation of very low density, the interference phenomena would most likely be different."

Einstein: "It probably wouldn't be as difficult to incorporate the interference phenomena as one thinks, and the reasons for this are as follows: it must not be assumed that radiations consist of non-interacting quanta; this would make it impossible to explain the phenomena of interference. I picture a quantum as a singularity surrounded by a large vector field. By using a large number of quanta one can construct a vector field that does not differ much from the kind of vector field we assume to be involved in radiations. I can well imagine that when rays impinge upon a boundary surface, a separation of the quanta takes place, due to interaction at the boundary surface, possibly according to the phase of the resulting field at which the quanta reach the interface. The equations for the resulting field would probably not be very different from those in the prevailing theory. It might not be necessary, with respect to interference, to change much in the currently prevailing conceptions. I would like to compare this with the process of molecularization of the carriers of the electrostatic field. The field, as brought about by atomized electric particles, is essentially not very different from the previous conceptions, and it is not out of the question that something similar will happen in the theory of radiation. I do not see any fundamental difficulty in the interference phenomena."

\section{Physical model of the photon}

If Einstein had produced a physical model of the photon that agreed with experiment he could have successfully answered Planck's objections and the foundations of quantum mechanics would perhaps appear differently today. Instead Planck's view, that photons have dual natures, prevails and it remains an open question whether a purely particle model is possible. To test Einstein's hypothesis we propose a photon model that consists of a localized concentration of pure field. 


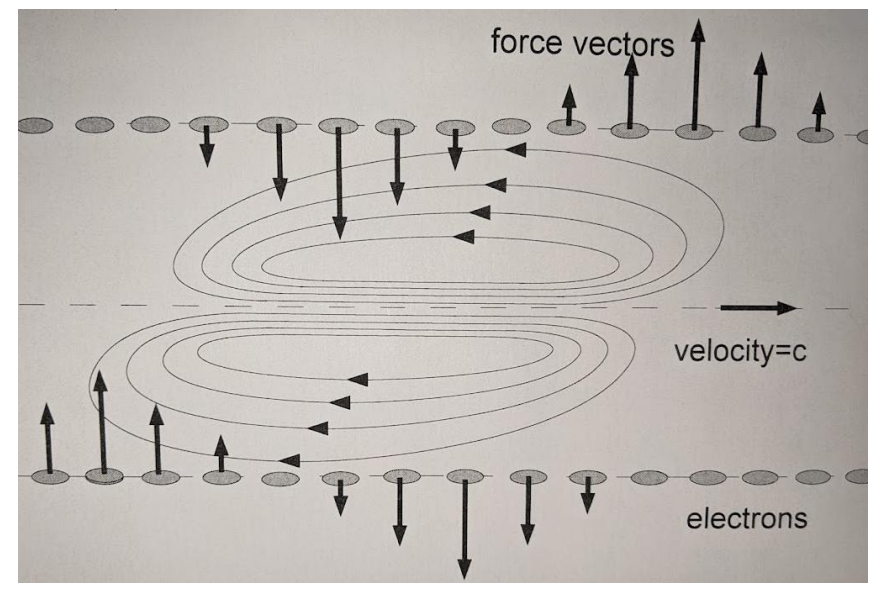

If it were possible to observe the photon by traveling at speed $\mathrm{c}$ its longitudinal velocity and the transverse sinusoidal oscillation of its electric and magnetic fields would necessarily cease, its proper time would equal zero, and we would see only static fields. A picture of an electromagnetic wave that complies with the conditions of both special relativity and classical theory, is localized, and is frozen in time is shown below. Thus the photon is conceived of as a localized concentration of $\mathbf{B}$ field whose poles are aligned with its motion and whose fields extend laterally in continuous loops to infinity. Transverse wave motion results when static magnetic potentials move past loosely bound negative charge centers. The interaction with electrons occurs without an exchange of energy because we know from experience that static magnetic fields do not contribute to the energy of charges in uniform relative motion. Force vectors are given by the right hand rule together with the Lorentz force equation $\mathbf{F}=\mathrm{q} / \mathrm{c}(\mathbf{v} X$ $\mathbf{B})$, where $\mathbf{v}=\mathbf{c}$ and reversals of $\mathbf{E}$ and $\mathbf{B}$ fields in electromagnetic waves are automatically determined by the vector product ( $\mathbf{v}$ X B).

The motion of a static magnetic field whose poles are aligned with its motion reproduces Maxwell's equations. All possible electromagnetic wave forms can be reproduced by the linear superposition of photons with field configurations of this type. Perhaps the most satisfying improvement made by the Einstein inspired vector field model as opposed to the electromagnetic wave picture of Maxwell is the natural elimination of fictitious displacement currents as unnecessary artifices. It also allows the core belief of quantum theory, waveparticle duality, to be interpreted classically by means of field geometry and vector addition alone. Diffuse external fields cause wave behavior to occur while the concentrated fields at the photon's core cause particle behavior. Thus interference and diffraction phenomena are caused by the superposition of photon fields precisely as Einstein envisioned. As a result of the model's inherent clarity we propose the use of fields; through cancellation, reinforcement, and superposition; as possible points of departure for all theories of nature in the hopes that 
complex mathematical models in other areas will be found to have simpler, classical explanations $[6,7,8,9]$.

\section{References}

[1] A. Einstein, "On the development of our views concerning the nature and constitution of radiation”, $\quad$ Phys $\quad Z \quad \mathbf{1 0}$ (1909): $817-825 \quad$ (Doc 60) https://einsteinpapers.press.princeton.edu/vol2-trans/

[2] A. Einstein, "On the electrodynamics of moving bodies" Ann Phys 17 (1905). (Doc 23) https://einsteinpapers.press.princeton.edu/vol2-trans/

[3] A. Einstein, "Does the inertia of a body depend on its energy content?", Ann Phys 18 (1905). (Doc 24) https://einsteinpapers.press.princeton.edu/vol2-trans/

[4] A. Einstein, "On a heuristic point of view concerning the production and transformation of light”, Ann Phys 17 (1905). (Doc14) https://einsteinpapers.press.princeton.edu/vol2trans/

[5] Discussion, Phys $Z \quad 10 \quad$ (1909), $\quad$ p. 825-6. $\quad$ (Doc 61) https://einsteinpapers.press.princeton.edu/vol2-trans/

[6] R. Oldani (IntechOpen, London, 2019). http://mts.intechopen.com/articles/show/title/lagrangian-quantum-mechanics-afully-relativistic-theory-of-atomic-structure

[7] R. Oldani (Intechopen, London, 2021) https://www.intechopen.com/books/quantum-chromodynamic/application-ofeinstein-s-methods-in-a-quantum-theory-of-radiation

[8] R. Oldani, Phys Essays "Einstein on measurement theory: A heuristic point of view" (March 2022), in print

[9] R. Oldani, Phys Essays “Quantum gravity for dummies”, 341 (2021). 